\title{
Expression and Clinical Significance of Tumor Markers CEA and CYFRA21-1 in Patients with Lung Cancer
}

\author{
H. F. ZHANG, G. LI'1, YONG XIA LIU², YA JIE WANG ${ }^{3 *}$ \\ Department of Laboratory Medicine, Beijing xicheng District Ping'an Hospital, No. 169 Zhaodengyu Road, Beijing 100035, \\ ${ }^{1}$ Department of Pathology, Beijing Tiantan Hospital, Capital Medical University, No 119 South 4 Round West Road, Beijing \\ 100070, ${ }^{2}$ Department of Laboratory Medicine, Beijing xicheng Zhanlan Road Hospital, No. 16, Taoliuyuan West Alley, Beijing \\ 100044, 32Department of Laboratory Medicine, Beijing Ditan Hospital, Capital Medical University, No. 8 Jingshun East Street, \\ Beijing 100015, China
}

\section{Zhang et al.: Significance of Tumor Markers CEA and CYFRA21-1 in Lung Cancer Patients}

\begin{abstract}
To explore the application value of tumor markers carcinoembryonic antigen and cytokeratin 19 fragment in the progression and prognosis of middle-advanced non-small cell lung cancer. Clinical data of 97 patients initially diagnosed with lung lesions in our hospital from January 2018 to December 2019 were collected and divided into the lung cancer group and benign lesion group. There were 52 patients diagnosed with stage I or II lung cancer by computed tomography and clinical diagnosis in the lung cancer group and 45 patients with benign lesions in the benign lesion group. The levels of serum tumor markers were detected by electrochemiluminescence immunoassay. The results showed that the levels of carcinoembryonic antigen and cytokeratin 19 were significantly higher in the lung cancer group compared with the benign lesion group and the difference was statistically significant $(p<0.05)$. Both carcinoembryonic antigen and cytokeratin 19 can be used in the diagnosis of squamous cell carcinoma and adenocarcinoma. The sensitivity of the combined detection was significantly higher than that of the single detection, while the specificity of the combined detection was significantly lower than that of carcinoembryonic antigen alone $(\mathbf{p}<0.05)$. In lung cancer with positive combined detection, the proportion of adenocarcinoma was significantly higher than that of squamous cell carcinoma. When cytokeratin 19 was positive and carcinoembryonic antigen was negative, the proportion of squamous cell carcinoma was significantly higher than that of adenocarcinoma. Serum carcinoembryonic antigen and cytokeratin 19 were abnormally highly expressed in non-Small cell lung cancer patients, which are useful for clinical diagnosing, treatment planning, efficacy monitoring, and prognosis evaluation.
\end{abstract}

Key words: Non-small cell lung cancer, serum carcinoembryonic antigen, cytokeratin 19 fragment, clinical significance

Non-Small Cell Lung Cancer (NSCLC) was one of the most common malignant tumors in the clinic, with high morbidity and mortality, accounting for about $80 \%$ of the total number of lung cancer in China. Because of its high malignant degree and poor prognosis, NSCLC was a difficult problem for scientists ${ }^{[1-3]}$. The incidence of NSCLC was relatively hidden, the early symptoms were not obvious, the diagnosis of patients was often in the middle-advanced stage. Clinically, the comprehensive treatment regimen based on chemotherapy was mainly adopted. Whether in the early diagnosis of tumor, in the monitoring of metastasis and recurrence or in the evaluation of efficacy and prognosis, tumor markers were very important clinical reference indicators ${ }^{[4,5]}$. Compared with the clinical monitoring techniques of Computed tomography (CT),
Positron emission tomography computed tomography (PET-CT), and specific biomarkers, the detection of serum tumor markers was more simple, rapid and less invasive and has important clinical application value $\mathrm{e}^{[6-9]}$. Many studies showed that the carcinoem-bryonic antigen (CEA) and human cytokeratin fragment antigen 21-1 (CYFRA21-1) has a high value in predicting tumor recurrence and judging the prognosis of patients $^{[2,3]}$. It was reported that different tumor markers have different specificity and sensitivity in different pathological types of tumors. This study retrospectively analyzed the changes of serum CEA and CYFRA21-1 levels in patients with middle-advanced stage NSCLC, to provide a reference for the disease progression and prognosis of NSCLC. The clinical data of 97 patients with lung lesions diagnosed in our hospital from

*Address for correspondence 
January 2018 to December 2019 were collected. Inclusion criteria includes Age $\leq 70$ years old; the patients were tested for serum CEA, CA125, and CYFRA21-1 voluntarily and regularly; Karnofsky performance status score (KPS score) $\geq 70$; normal function of heart, liver, kidney, and other important organs and normal hematopoietic function. Exclusion criteria includes patients with other malignant tumors; patients with severe heart, liver, kidney, and other organ dysfunction; patients with a history of anti-tumor therapy; patients who received radiotherapy at the same time during treatment; patients with hematological and immune system diseases; patients with brain metastasis; patients with poor treatment compliance and could not meet the standard treatment for 2 weeks. Finally, 52 patients with stage I or II lung cancer diagnosed by $\mathrm{CT}$ and clinical diagnosis were included in the lung cancer group (LC group), while 45 patients with benign lesions diagnosed by $\mathrm{CT}$ and clinical diagnosis were included in the benign disease group (BLD group). In the LC group, the age of the patients ranged from 26-68 $y$ old, with an average age of $63.20 \pm 6.40 \mathrm{y}$ old, including 34 males and 18 females. According to the pathological types, they were divided into squamous cell carcinoma $(\mathrm{n}=22)$, adenocarcinoma $(\mathrm{n}=26)$ and large cell lung cancer $(n=4)$. The age of BLD group was $22-67 \mathrm{y}$ old, and the average age was $62.80 \pm 5.70 \mathrm{y}$ old. There were 31 males and 14 females. According to the pathological types, they were divided into pure lung infection $(n=26)$, benign lung nodules $(n=6)$, tuberculosis $(n=9)$ and grinders' disease $(n=4)$. Statistical analysis showed that there was no significant difference in the age and sex ratio between the two groups $(p>0.05)$. The serum of all patients before treatment was collected. Five $\mathrm{ml}$ of fasting blood was collected in the morning, and the serum was separated after centrifugation for detection. The fully automatic electrochemiluminescence immunoanalyzer E602 and its original matching reagents (Roche) were used for detection. The positive results of serum tumor markers were $C E A \geq 3.4 \mathrm{ng} / \mathrm{ml}$, CYFRA21-1 $\geq 3.3 \mathrm{ng} / \mathrm{ml}$. SPSS 20.0 was used for statistical analysis. The measurement data belonged to skewed distribution by normal distribution test, and Mann-Whitney U test was used to analyze. The counting data were expressed as percentage, and $\chi^{2}$ test was used to analyze. $p<0.05$ indicates that the difference is statistically significant. The results showed that compared with BLD group, the serum levels of two tumor markers in LC group were significantly higher than those in LC group ( $p<0.05$, fig. 1). There was no significant difference in the expression of CEA and
CYFRA21-1 between adenocarcinoma and squamous cell carcinoma in LC group ( $>0.05$, fig. 2 ). The results showed that the positive rates of two tumor markers in the serum of LC patients were significantly higher than those of BLD group. At the same time, in the sensitivity test, the result of the combined detection was significantly higher than that of the single index detection, and the difference was statistically significant $(p<0.05)$. In the specificity test, the result of the combined detection was significantly higher than that of CEA alone and there was no significant difference between the combined detection and CYFRA21-1 alone ( $>0.05$, fig. 3 and 4 ). There was no significant difference in the positive rate of CEA and CYFRA21-1 between adenocarcinoma and squamous cell carcinoma in the LC group ( $p>0.05$, fig. 5). When the serum markers CEA and CYFRA21-1 were both positive in

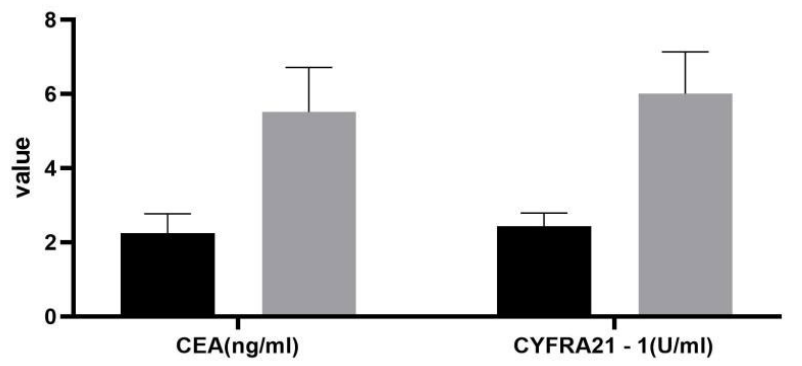

Fig. 1: Comparison of tumor markers between the two groups (口): BLD, ( $)$ : LC

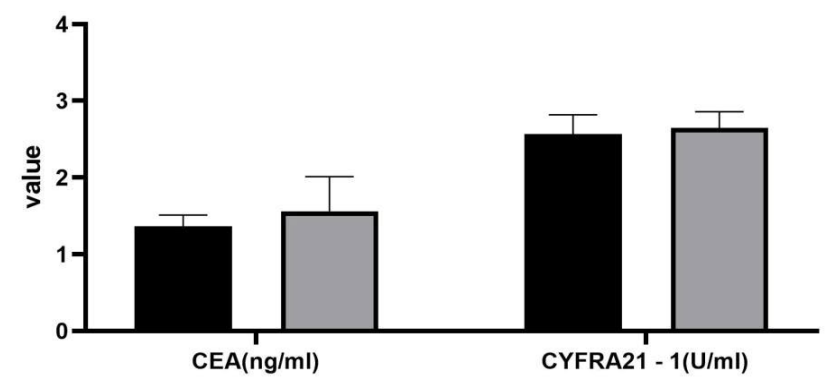

Fig. 2: The expression of CEA and CYFRA21-1 between adenocarcinoma and squamous cell carcinoma ( $\square$ ): Adenocarcinoma, (

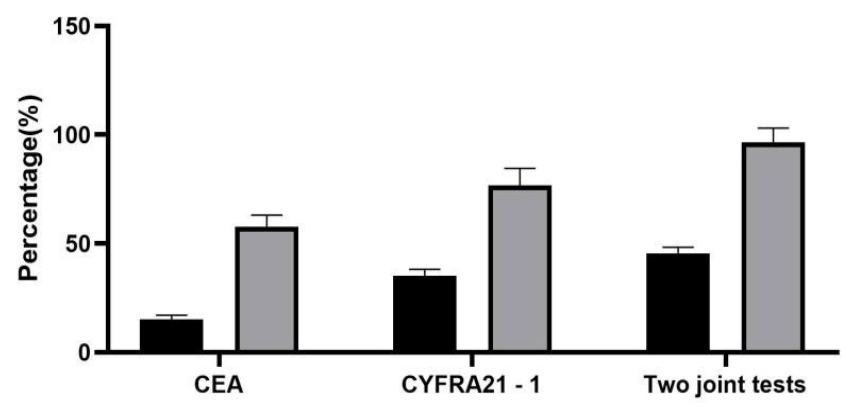

Fig. 3: Sensitivity of two tumor markers ( $\square)$ : BLD, (口): LC 


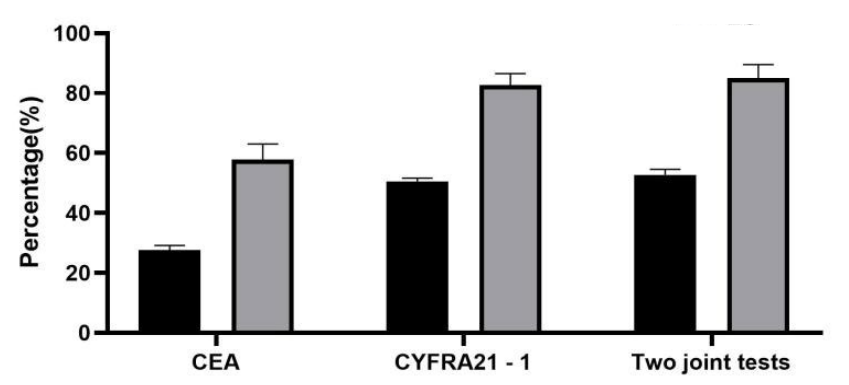

Fig. 4: Specificity of two tumor markers (口): LC

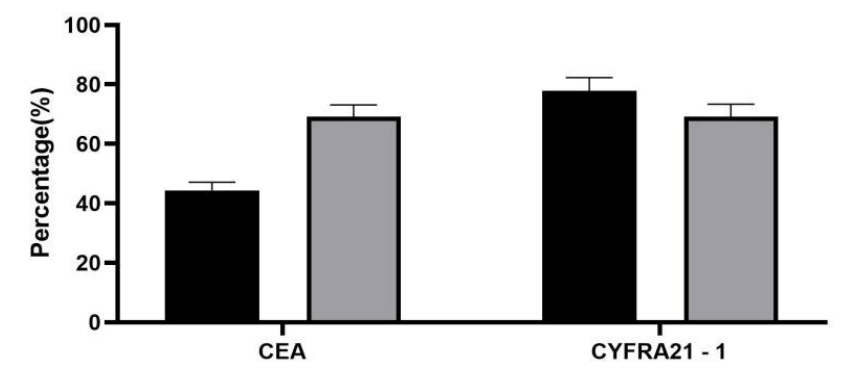

Fig. 5: Positive rate of CEA and CYFRA21-1 in adenocarcinoma and squamous cell carcinoma ( $\square$ ): Adenocarcinoma, (ए): Squamous cell carcinoma

patients with lung cancer, the proportion of adenocarcinoma was $100 \%$, which was significantly higher than that in other tissue types of cancer $(p<0.05)$. When CYFRA21-1 was positive and CEA was negative, the proportion of squamous cell carcinoma was significantly higher than that of adenocarcinoma $(p<0.05)$, as shown in Table 1 . NSCLC was a solid tumor based on neovascularization, accounting for about $79 \%$ of the primary tumors ${ }^{[2]}$. Compared with the traditional invasive and imaging detection, the detection of serum tumor markers has been widely concerned in the clinic because of its advantages such as lower cost, less trauma, and simple operation, which was helpful to the diagnosis and evaluation of tumor disease. CEA was highly expressed in the lung cancer ${ }^{[4-5]}$, It was often used in the early diagnosis, prognosis, and curative effect evaluation of lung cancer. CYFRA21-1 was a tumor marker of NSCLC, especially for squamous cell carcinoma $^{[6]}$. The expression of CEA and CYFRA21-1 was positively correlated with the clinical stage of lung cancer. If we take all stages of lung cancer as the object of study to explore the diagnostic value of CEA and CYFRA21-1, the false positive rate of sensitivity and specificity may be increase ${ }^{[10,11]}$. For the diagnostic methods of early lung cancer, such as imaging, pathology, tumor markers, the information obtained by an isolated diagnostic method was limited ${ }^{[12]}$. From the point of view of the combined application of various methods in clinical practice and solving the difficulties in diagnosis, the diagnostic value of tumor markers in early lung cancer can be evaluated more objectively and practically. We detected the expression of CEA and CYFRA21-1 in the serum of 52 patients with early lung cancer and 45 patients with benign pulmonary lesions. The results showed that the expression level of CEA and CYFRA21-1 in the lung cancer patients was significantly higher than that of patients with benign pulmonary lesions. The positive rate of the combined detection of CEA and CYFRA21-1 was higher than that of single detection, indicating that the combined detection can improve the sensitivity of early lung cancer detection significantly, and has high clinical value for the detection and diagnosis of early lung cancer. However, because the specificity of the combined test was lower than that of the single detection, a comprehensive analysis of two indexes was still needed in the definite diagnosis, and the diagnosis should be combined imaging and pathological examination. This study found that there was no significant difference in the expression of serum CEA and CYFRA21-1 between adenocarcinoma and squamous cell carcinoma. Tumor markers have more reference value in the early lung cancer where imaging and pathology were not yet clear, which was in line with the urgent need for early diagnosis of lung cancer and clinical application of tumor markers. If more patients with advanced lung cancer and patients after treatment were included in the experiment, there may be significant differences in the expression levels of CYFRA21-1 and CEA between patients with adenocarcinoma and squamous cell carcinoma. Similarly, this experiment shows that the serum levels of the three markers have a skewed distribution in the early stage of lung cancer, but it does not rule out the possibility that the above three markers have a normal distribution in the development of advanced lung cancer or in the whole process of lung cancer. In this study, it was not found that CYFRA21-1 was highly sensitive to

\section{TABLE 1: DETECTION OF DIFFERENT PATHOLOGICAL TYPES OF CANCER BY THE COMBINATION OF MARKERS}

\begin{tabular}{lcc}
\hline Combined detection & $\begin{array}{c}\text { CEA(+), } \\
\text { CYFRA21-1(+) } \\
(\%)\end{array}$ & $\begin{array}{c}\text { CEA(-), } \\
\text { CYFRA21-1(+) } \\
(\%)\end{array}$ \\
\hline $\begin{array}{l}\text { Squamous cell } \\
\text { carcinoma ( } \mathrm{\%}=22)\end{array}$ & $0(0.00)$ & $10(45.45)$ \\
$\begin{array}{l}\text { Adenocarcinoma (n=26) } \\
\text { Large cell lung cancer } \\
(\mathrm{n}=4)\end{array}$ & $26(100.0)$ & $4(15.38)$ \\
$\mathrm{x}^{2}$ & $0(0.00)$ & $0(0.00)$ \\
$\mathrm{p}$ & 12.55 & 5.81 \\
\hline
\end{tabular}


squamous cell carcinoma. In the early and advanced stages of lung cancer, the sensitivity of CYFRA21-1 to the squamous cell carcinoma may be significantly different. And the sensitivity in the early stage was poor, it does not rule out the high sensitivity in the late stage of lung cancer.

In this study, it was found that the specificity of the combined detection of CEA and CYFRA21-1 was lower than that of single detection, but not that there was no significant difference reported in most literatures ${ }^{[13-15]}$. We chose the patients who were clinically diagnosed as benign lesions included in the BLD group, which was consistent with the clinical application requirements of using tumor markers to determine the diagnosis of lung cancer. When we study the value of tumor markers in the diagnosis of lung cancer, we take the benign lesions which were easy to be confused with lung cancer as a reference. In the clinical work, we also found that the tumor markers of benign lung lesions which were easy to be diagnosed often do not exceed the reference range. To sum up, serum CEA and CYFRA21-1 were abnormally high expressed in patients with NSCLC, which is useful for clinical diagnosing, treatment planning, efficacy monitoring, and prognosis evaluation.

\section{Acknowledgements:}

This work was supported Beijing xicheng District Ping'an Hospital, Beijing Tiantan Hospital, Capital Medical University, Beijing xicheng Zhanlan Road Hospital and Beijing Ditan Hospital, Capital Medical University.

\section{Declaration of interest:}

The authors report no conflicts of interest.

\section{REFERENCES}

1. Egger M, Davey Smith G, Schneider M, Minder C. Bias in meta-analysis detected by a simple, graphical test. BMJ 1997;315:629-34.

2. Barak V, Holdenrieder S, Nisman B, Stieber P. Relevance of circulating biomarkers for the therapy monitoring and followup investigations in patients with non-small cell lung cancer. Cancer Biomark 2010;6:191-6.

3. Glas AS, Lijmer JG, Prins MH, Bonsel GJ, Bossuyt PM. The diagnostic odds ratio: a single indicator of test performance. $\mathrm{J}$ Clin Epidemiol 2003;56:1129-35.

4. Ardizzoni A, Cafferata MA, Tiseo M, Filiberti R, Marroni P,
Grossi $\mathrm{F}$, et al. Decline in the serum carcinoembryonic antigen and cytokeratin 19 fragment during chemotherapy predicts objective response and survival in patients with advanced nonsmall cell lung cancer. Cancer 2006;107:2842-9.

5. Arrieta O, Villarreal-Garza C, Martínez-Barrera L, Morales M, Dorantes-Gallareta Y, Peña-Curiel O, et al. Usefulness of serum carcinoembryonic antigen (CEA) in evaluating response to chemotherapy in patients with advanced non-small-cell lung cancer: a prospective cohort study. BMC Cancer 2013;13:254.

6. Gridelli C, Rossi A, Maione P. Treatment of non-small-cell lung cancer: state of the art and development of new biologic agents. Oncogene 2003;22:6629-38.

7. Merle P, Janicot H, Filaire M, Roux D, Bailly C, Vincent C, et al. Early CYFRA 21-1 variation predicts tumor response to chemotherapy and survival in locally advanced non-small cell lung cancer patients. Int J Biol Markers 2004;19:310-5.

8. Nisman B, Biran H, Heching N, Barak V, Ramu N, Nemirovsky I, et al. Prognostic role of serum cytokeratin 19 fragments in advanced non-small-cell lung cancer: association of marker changes after two chemotherapy cycles with different measures of clinical response and survival. Br J Cancer 2008;98:77-9.

9. Wang J, Yi Y, Li B, Wang Z, Sun H, Zhang P, et al. CYFRA21-1 can predict the sensitivity to chemoradiotherapy of non-smallcell lung carcinoma. Biomarkers 2010;15:594-601.

10. Molina R, Marrades RM, Auge JM, Escudero JM, Viñolas N, Reguart $\mathrm{N}$, et al. Assessment of a combined panel of six serum tumor markers for lung cancer. Am J Respir Crit Care Med 2016;193:427-37.

11. Walter SD. Properties of the summary receiver operating characteristic (SROC) curve for diagnostic test data. Stat Med 2002;21:1237-56.

12. Zhi XY, Yu JM, Shi YK. Chinese guidelines on the diagnosis and treatment of primary lung cancer (2015 version). Cancer 2015;121:3165-81.

13. Jin B, Huang AM, Zhong RB, Han BH. The value of tumor markers in evaluating chemotherapy response and prognosis in Chinese patients with advanced non-small cell lung cancer. Chemotherapy 2010;56:417-23.

14. Liu H, Gu X, Lv T, Wu Y, Xiao Y, Yuan D, et al. The role of serum carcinoembryonic antigen in predicting responses to chemotherapy and survival in patients with non-small cell lung cancer. J Cancer Res Ther 2014;10:239-43.

15. Vollmer RT, Govindan R, Graziano SL, Gamble G, Garst J, Kelley MJ, et al. Serum CYFRA 21-1 in advanced stage nonsmall cell lung cancer: an early measure of response. Clin Cancer Res 2003;9:1728-33.

This is an open access article distributed under the terms of the Creative Commons Attribution-NonCommercial-ShareAlike 3.0 License, which allows others to remix, tweak, and build upon the work non-commercially, as long as the author is credited and the new creations are licensed under the identical terms

This article was originally published in a special issue, "Trends in Therapeutic Management of Various Clinical Conditions II" Indian J Pharm Sci 2021:83(2)Spl issue;110-113 